\title{
openheart Natriuretic peptides for the detection of paroxysmal atrial fibrillation
}

\author{
Joachim Seegers, ${ }^{1,2}$ Markus Zabel, ${ }^{1}$ Timo Grüter, ${ }^{1}$ Antje Ammermann, ${ }^{3}$ \\ Mark Weber-Krüger, ${ }^{1}$ Frank Edelmann, ${ }^{1,4,5,6}$ Götz Gelbrich, ${ }^{7}$ Lutz Binder, ${ }^{8}$ \\ Christoph Herrmann-Lingen, ${ }^{4,9}$ Klaus Gröschel, ${ }^{10}$ Gerd Hasenfuß, ${ }^{1,4}$ \\ Nicolas Feltgen, ${ }^{3}$ Burkert Pieske, ${ }^{5,6}$ Rolf Wachter ${ }^{1,4}$
}

To cite: Seegers J, Zabel M, Grüter T, et al. Natriuretic peptides for the detection of paroxysmal atrial fibrillation. Open Heart 2015;2:e000182. doi:10.1136/openhrt-2014000182

Received 8 August 2014 Revised 18 November 2014 Accepted 20 January 2015

\section{CrossMark}

For numbered affiliations see end of article.

Correspondence to Dr Rolf Wachter; Wachter@med.unigoettingen.de

\section{ABSTRACT}

Background and purpose: Silent atrial fibrillation (AF) and tachycardia (AT) are considered precursors of ischaemic stroke. Therefore, detection of paroxysmal atrial rhythm disorders is highly relevant, but is clinically challenging. We aimed to evaluate the diagnostic value of natriuretic peptide levels in the detection of paroxysmal AT/AF in a pilot study.

Methods: Natriuretic peptide levels were analysed in two independent patient cohorts (162 patients with arterial hypertension or other cardiovascular risk factors and 82 patients with retinal vessel disease). Nterminal-pro-brain natriuretic peptide (NT-proBNP) and BNP were measured before the start of a 7-day Holter monitoring period carefully screened for AT/AF.

Results: 244 patients were included; 16 had paroxysmal AT/AF. After excluding patients with a history of $A T / A F(n=5), 14$ patients had newly diagnosed AT/AF (5.8\%) NT-proBNP and BNP levels were higher in patients with paroxysmal AT/AF in both cohorts: (1) 154.4 (IQR 41.7; 303.6) versus 52.8 $(30.4 ; 178.0) \mathrm{pg} / \mathrm{mL}$ and $70.0(31.9 ; 142.4)$ versus $43.9(16.3 ; 95.2)$ and (2) $216.9(201.4 ; 277.1)$ versus $90.8(42.3-141.7)$ and $96.0(54.7 ; 108.2)$ versus 29.1 (12.0; 58.1). For the detection of AT/AF episodes, NTproBNP and BNP had an area under the curve in receiver operating characteristic analysis of $0.76(95 \%$ $\mathrm{Cl}, 0.64$ to $0.88 ; \mathrm{p}=0.002)$ and 0.75 (0.61 to 0.89 ; $p=0.004$ ), respectively.

Conclusions: NT-proBNP and BNP levels are elevated in patients with silent AT/AF as compared with sinus rhythm. Thus, screening for undiagnosed paroxysmal AF using natriuretic peptide level initiated Holter monitoring may be a useful strategy in prevention of stroke or systemic embolism.

\section{INTRODUCTION}

Atrial fibrillation (AF) is an established risk factor for ischaemic stroke. ${ }^{1}$ A meta-analysis indicates that oral anticoagulation therapy with warfarin reduces stroke risk by $64 \%$ on an intention to treat basis. ${ }^{2}$ Identifying paroxysmal AF may be challenging in patients presenting with sinus rhythm, especially as episodes of AF may not be symptom related, ${ }^{3}$

\section{KEY MESSAGES}

What is already known about this subject? The early detection of paroxysmal atrial fibrillation (AF) is a very attractive strategy for primary stroke prevention. Extensive Holter monitoring up to 7 days is useful to detect AF, but it is not feasible to screen the whole population.

How might this impact on clinical practice? A biomarker screening to decide whether or not to perform extended Holter monitoring for the first detection on AF may reduce the number of patients to be screened by Holter. Once AF is detected, $>90 \%$ of patients need oral anticoagulants for primary stroke prevention. Biomarker level-guided indication of Holter ECG may be an attractive strategy for AF detection and thus primary stroke prevention.

What does this study add?

Brain natriuretic peptide and N-terminal-pro-BNP values in patients with paroxysmal AF were significantly higher and showed very good diagnostic properties for AF detection. Therefore, these markers may be very useful for identifying patients with otherwise undetected AF.

but the risk for thromboembolism is similar to that for permanent $\mathrm{AF}^{4}$ Prolonged, ${ }^{5}{ }^{6}$ or continuous, $^{7}$ rhythm monitoring may enhance the detection of this clinically 'silent' paroxysmal AF. Although such extended diagnostics have been performed in a large number of patients, ${ }^{8}$ this seems to be not feasible for clinical routine as they are uncomfortable for the patient and timeconsuming. Investigation on natriuretic peptides has shown their potential to predict $\mathrm{AF}$ within the general population, ${ }^{9}$ as well as in patients undergoing surgical procedures. ${ }^{10}$ Moreover, elevation of natriuretic peptide levels was shown in patients suffering from $\mathrm{AF}^{11}{ }^{12}$ which decreases after conversion to sinus rhythm. ${ }^{13} 14$ Recently, we have shown that the brain natriuretic peptide (BNP) is 
an independent predictor of paroxysmal AF detected by prolonged ECG monitoring in patients with cerebral ischaemia (secondary stroke prevention), ${ }^{15}$ Thus, we hypothesised that natriuretic peptide levels may also help to identify $\mathrm{AF}$ in patients with cardiovascular risk factors as a potential prevention strategy and aimed to evaluate the diagnostic value in a pilot study.

\section{MATERIALS AND METHODS Patients}

Patients participating in this observational trial were recruited from the collective of the ongoing noninterventional diastolic congestive heart failure (Diast-CHF) trial, which is part of the nationwide German Competence Network Heart Failure. Inclusion and exclusion criteria have been described previously. ${ }^{16}$ Briefly, a total of 1735 patients with at least one risk factor for heart failure with preserved left ventricular function (defined as history of hypertension, diabetes mellitus, sleep apnoea syndrome or atherosclerotic disease) or established CHF were referred by a network of primary care physicians between 2004 and 2006. After the baseline visit which included N-terminal-pro-brain natriuretic peptide (NT-proBNP) analysis, NT-proBNP values were divided into quartiles. Only patients with NT-proBNP in the lowest (NT-proBNP $<53 \mathrm{pg} / \mathrm{mL}$ ) and in the highest NT-proBNP-quartile (NT-proBNP $>226 \mathrm{pg} / \mathrm{mL}$ ) at the baseline visit presenting with sinus rhythm in the 12-lead ECG and without an implanted cardiac device were selected and randomly asked to participate in this substudy which was part of the 1 year follow-up examination of the patients. Patient selection and all clinical examinations were performed by investigators blinded to NT-proBNP values and quartiles. As systolic dysfunction (ejection fraction $<50 \%$ ) is a known cause of elevated natriuretic peptide levels, these patients were excluded. Diast-CHF complies with the Declaration of Helsinki; the protocol was approved by the responsible ethics committee and all patients gave written informed consent. The recruitment process of the Diast-CHF substudy is shown in figure 1.

In addition, a second cohort was added using data from patients enrolled in the Find-AF eye study. In brief, Find-AF eye is a single centre study and consecutively enrolled 101 patients with retinal artery occlusion or venous retinal thrombosis. Only patients free of $\mathrm{AF}$ on admission ECG and with an evaluable 7-day Holter ECG were included in the analysis of this manuscript $(n=82)$.

\section{Data collection and clinical evaluation}

All patients underwent medical history, physical examination, a 12-lead ECG and standard echocardiography at baseline.

\section{Blood analysis}

Blood samples were drawn immediately before Holter ECG was started and were stored at $-80^{\circ} \mathrm{C}$ for later



Figure 1 Flow chart of the recruitment process (diastolic congestive heart failure, Diast-CHF; NT-proBNP, $\mathrm{N}$-terminal-pro-brain natriuretic peptide).

analysis. Plasma levels of NT-proBNP were measured using a sandwich immunoassay (Roche Diagnostics, Mannheim, Germany). BNP was measured by means of a sandwich immunoassay (Abbott GmbH \& Co. KG, Wiesbaden, Germany). N-terminal pro atrial natriuretic peptide (NT-proANP) was measured by using a sandwich immunoassay (Biomedica Medizinprodukte, Vienna, Austria). Personnel responsible for the determination of natriuretic peptide levels were blinded to clinical patient data.

\section{Holter ECG}

A dual-channel Holter ECG was recorded with digital portable recorders (Diast-CHF: Lifecard CF, Del Mar Reynolds Medical Ltd, Hertford, UK, and Find-AF eye: getemed, Teltow, Germany) for seven consecutive days. Analysis was performed with Pathfinder digital (Software Version V8.602, Del Mar Reynolds Medical Ltd) or Cardioday (getemed). Episodes with sudden rapid changes in heart rate or sudden onset of arrhythmia in the manual review of the Holter ECG were carefully analysed for AF and atrial flutter (atrial tachycardia, AT). $\mathrm{AT} / \mathrm{AF}$ was diagnosed if episodes lasted more than $30 \mathrm{~s}$. 
Equivocal findings were cograded by an additional physician blinded to the data.

\section{Definition of primary end point}

The primary hypothesis was that higher natriuretic peptide levels can discriminate patients with paroxysmal $\mathrm{AT} / \mathrm{AF}$ from those without AT/AF. We measured both NT-proBNP and BNP (which is measured in many institutions instead of NT-proBNP).

\section{Statistical analyses}

Continuous data are shown as mean \pm SD. Values of natriuretic peptides were not normally distributed and are therefore given as median and IQR of 25th and 75th centile. Categorical variables are given as absolute numbers (percentage). Receiver operating characteristic (ROC) curves were used to describe test characteristics. Statistical tests were performed with SPSS Statistics 21.0 (IBM, Chicago, Illinois, USA). A p value below 0.05 was considered to be significant.

\section{RESULTS}

\section{Study population}

Of the patients included in the Diast-CHF trial, a total of 615 patients were eligible in principle; the number of Holter recorder systems and the analysis time available was the most relevant restriction to patient recruitment. Of the 200 patients asked to participate, 167 provided written informed consent. Five patients were excluded for technical problems during the Holter recording (figure 1). Of the remaining 162 patients, $156(96 \%)$ had 7 days of recording, whereas the other 6 patients had at least 4 days of recording. The Find-AF eye cohort consisted of 82 patients with an evaluable 7 day Holter ECG.

The baseline characteristics of the 162 patients of the Diast-CHF substudy and the additional 82 patients taken from the Find-AF eye study are displayed in table 1. Five (2\%) patients with a history of AF in the Diast-CHF cohort were excluded for further analysis. In both cohorts combined, $14(5.8 \%)$ patients had AT/AF for at least $30 \mathrm{~s}$ during Holter monitoring (table 2). Median duration (IQR) of AT/AF was 24.5 (3.3-1046.8) min, and eight patients had an episode of at least $6 \mathrm{~min}$ (ASSERT criteria). ${ }^{17}$

\section{Natriuretic peptides and paroxysmal AF}

Table 3 shows the median and IQR of BNP and NT-proBNP levels. NT-proANP was only measured in Find-AF eye. Plasma levels of natriuretic peptides were numerically higher in paroxysmal $\mathrm{AT} / \mathrm{AF}$ patients in both cohorts.

\section{Diagnostic utility of natriuretic peptides}

For ROC analysis, data of both cohorts were pooled to determine the diagnostic utility of natriuretic peptides for the detection of paroxysmal AT/AF. Figure 2 shows the ROC curves for BNP and NT-proBNP; area under curve was 0.75 (95\% CI, 0.61 to $0.89 ; \mathrm{p}=0.004)$ and 0.76 (95\% CI, 0.64 to 0.88 ; $\mathrm{p}=0.002$ ), respectively.

\section{Clinical relevance}

The finding of paroxysmal AF in our study was of clinical relevance. In 13 of the 14 patients, the detection of paroxysmal AF would have led to a change in antithrombotic therapy according to recent guidelines ${ }^{18}$ (from no therapy to oral anticoagulation in 5 patients and from aspirin to oral anticoagulation in 8 patients).

\section{DISCUSSION}

The major finding of this study is that natriuretic peptide plasma levels are higher in patients suffering from paroxysmal AT/AF compared with those without $\mathrm{AT} / \mathrm{AF}$ in two different independent cohorts. Although natriuretic peptides are mainly used for the diagnosis of heart failure, ${ }^{19}$ they are also elevated in a number of different diseases (eg, asymptomatic systolic and diastolic dysfunction, ${ }^{20}$ pulmonary embolism ${ }^{21}$ and other diseases).

In addition, natriuretic peptides have also been shown to be elevated in patients with $\mathrm{AF}$ as compared to patients with sinus rhythm, ${ }^{14}$ Moreover, natriuretic peptides have been shown to predict future AF in several clinical settings, ${ }^{9} \quad 22$ Pathophysiological, increased haemodynamic load causes atrial stretch, which is well known as a reason for development of $\mathrm{AT} / \mathrm{AF}^{23}$ Natriuretic peptides correlate with haemodynamic load; ${ }^{19}$ thus, their indicative value for diagnosis of unknown $\mathrm{AT} / \mathrm{AF}$ may be equal or superior to clinical risk factors of AF.

Recently, we showed that natriuretic peptides are elevated in patients with stroke with undiagnosed paroxysmal $\mathrm{AF}$ as compared with sinus rhythm. ${ }^{15}$ We now extend these findings to two different clinical settings: patients with risk factors for heart failure and patients with retinal vessel occlusion.

The clinical relevance of early detection of the so-called 'silent AT/AF' was recently underlined by the ASSERT study which revealed that patients with short episodes of AF ( $>6$ min within 3 months) have a 2.5 -fold increase in the risk of stroke and systemic embolism. ${ }^{17}$ The early detection of subclinical AF in patients at risk for $\mathrm{AF}$ is a major challenge and potentially a very effective stroke prevention strategy. ${ }^{17}{ }^{24}$ Recently, a stepwise screening approach was reported in elderly patients to identify (asymptomatic) paroxysmal AF. During a 2-week recording of 20-30 s two times per day using a handheld ECG in 75-year-old patients without a history of AF, paroxysmal AT/AF was identified in only $4 \%$, indicating that a large number of patients have to be screened to diagnose one patient with paroxysmal AT/AF. Natriuretic peptides were not used in this study for preselecting study participants. In daily life, risk of $\mathrm{AT} / \mathrm{AF}$ development are commonly determined first by clinical 
Table 1 Patient characteristics

\begin{tabular}{|c|c|c|c|c|}
\hline & \multicolumn{2}{|l|}{ DIAST-CHF } & \multicolumn{2}{|l|}{ Find-AF eye } \\
\hline & $\begin{array}{l}\text { No paroxysmal } \\
\text { AT/AF }(n=152)\end{array}$ & $\begin{array}{l}\text { Paroxysmal } \\
\text { AT/AF }(n=10)\end{array}$ & $\begin{array}{l}\text { No paroxysmal } \\
\text { AT/AF }(n=76)\end{array}$ & $\begin{array}{l}\text { Paroxysmal } \\
\text { AT/AF }(n=6)\end{array}$ \\
\hline Age (years) & $64 \pm 7$ & $66 \pm 7$ & $63 \pm 11$ & $77 \pm 6$ \\
\hline Male gender (\%) & $90(59)$ & $6(60)$ & $48(63)$ & $4(67)$ \\
\hline BMI $\left(\mathrm{kg} / \mathrm{m}^{2}\right)$ & $28.8 \pm 4.4$ & $32.4 \pm 4.0$ & $28.2 \pm 5.5$ & $28.8 \pm 6.2$ \\
\hline Heart rate (bpm) & $72 \pm 14$ & $71 \pm 13$ & $76 \pm 14$ & $68 \pm 15$ \\
\hline Systolic blood pressure (mm Hg) & $147 \pm 20$ & $148 \pm 19$ & $152 \pm 27$ & $127 \pm 9$ \\
\hline Diastolic blood pressure (mm Hg) & $84 \pm 12$ & $82 \pm 11$ & $88 \pm 17$ & $71 \pm 8$ \\
\hline \multicolumn{5}{|l|}{ Laboratory } \\
\hline Creatine $(\mathrm{mg} / \mathrm{dL})$ & $1.0 \pm 0.3$ & $0.9 \pm 0.2$ & $1.0 \pm 0.4$ & $1.4 \pm 0.7$ \\
\hline Haemoglobin (mg/dL) & $14.2 \pm 1.2$ & $13.8 \pm 1.1$ & $14.2 \pm 1.4$ & $14.2 \pm 1.6$ \\
\hline Thyroid-stimulating hormone (iU/mL) & $1.53 \pm 5.92$ & $1.67 \pm 0.80$ & $1.39 \pm 0.83$ & $1.27 \pm 0.60$ \\
\hline \multicolumn{5}{|l|}{ ECG } \\
\hline$P Q$ interval (ms) & $168 \pm 26$ & $180 \pm 31$ & $166 \pm 30$ & $185 \pm 39$ \\
\hline QRS duration (ms) & $92 \pm 13$ & $97 \pm 12$ & $91 \pm 14$ & $104 \pm 17$ \\
\hline QT interval (ms) & $389 \pm 33$ & $403 \pm 30$ & $379 \pm 31$ & $407 \pm 29$ \\
\hline \multicolumn{5}{|l|}{ Echo } \\
\hline Left atrial diameter (mm) & $41 \pm 5$ & $46 \pm 4$ & $42 \pm 4$ & $47 \pm 6$ \\
\hline Tissue Doppler a'-wave (cm/s) & $11.0 \pm 2.5$ & $9.7 \pm 2.1$ & $10.8 \pm 2.1$ & $3.6 \pm 0.4$ \\
\hline Left ventricular ejection fraction (\%) & $60 \pm 7$ & $60 \pm 8$ & $64 \pm 9$ & $64 \pm 8$ \\
\hline Left ventricular mass index $\left(\mathrm{g} / \mathrm{m}^{2}\right)$ & $117 \pm 24$ & $128 \pm 23$ & $94 \pm 27$ & $120 \pm 52$ \\
\hline \multicolumn{5}{|l|}{ Co-disease } \\
\hline Hypertension (\%) & $140(92)$ & $8(80)$ & 37 (49) & $5(83)$ \\
\hline Diabetes (\%) & $42(28)$ & $3(30)$ & $9(12)$ & $3(50)$ \\
\hline Smoker (\%) & $82(54)$ & $3(30)$ & $46(61)$ & $3(50)$ \\
\hline Hyperlipidaemia (\%) & $71(47)$ & $5(50)$ & $16(21)$ & $4(67)$ \\
\hline Coronary artery disease (\%) & $32(21)$ & $2(20)$ & $6(8)$ & $2(33)$ \\
\hline History of AF (\%) & $3(2)$ & $2(20)$ & $0(0)$ & $0(0)$ \\
\hline Prior stroke (\%) & $5(3)$ & $2(20)$ & $2(3)$ & $0(0)$ \\
\hline $\mathrm{CHA}_{2} \mathrm{DS}_{2} \mathrm{VASc}$ Score (median (IQR)) & $3(2 ; 3)$ & $3(1 ; 4)$ & $2(1 ; 2)$ & $4(4 ; 4)$ \\
\hline
\end{tabular}

$\mathrm{AF}$, atrial fibrillation; $\mathrm{AT}$, atrial tachycardia; BMI, body mass index; DIAST-CHF, diastolic congestive heart failure.

risk factors (eg, age) followed by a standard 12-lead ECG. However, these workups will identify a small number of patients with 'silent' AT/AF. ${ }^{25}$ Thus, if AT/
$\mathrm{AF}$ is assumed but not diagnosed, identifying those patients with an increased risk of paroxysmal AT/AF using natriuretic peptides seems highly attractive.

Table 2 AT/AF burden, prior antithrombotic therapy and $\mathrm{CHA}_{2} \mathrm{DS}_{2}-\mathrm{VASc}$ Score ${ }^{28}$ of the 14 patients with AT/AF detected by prolonged Holter monitoring

\begin{tabular}{|c|c|c|c|c|c|c|}
\hline & $\begin{array}{l}\text { Episodes } \\
\text { (n) }\end{array}$ & $\begin{array}{l}\text { Cumulative } \\
\text { time of } \\
\text { AT/AF (min) }\end{array}$ & $\begin{array}{l}\text { Type of } \\
\text { arrhythmia }\end{array}$ & $\begin{array}{l}\text { At least } \\
1 \text { episode } \\
>6 \text { min }\end{array}$ & $\begin{array}{l}\text { Antithrombotic/ } \\
\text { antiplatelet } \\
\text { therapy }\end{array}$ & $\begin{array}{l}\mathrm{CHA}_{2} \mathrm{DS}_{2}-\mathrm{VASc} \\
\text { score }\end{array}$ \\
\hline Diast-CHF \#2 & 3 & 2 & AT & No & None & 1 \\
\hline Diast-CHF \#4 & 1 & 2 & AF & No & ASA & 4 \\
\hline Diast-CHF \#5 & 4 & 7 & AT & No & None & 2 \\
\hline Diast-CHF \#6 & 1 & 1066 & AF & Yes & ASA & 8 \\
\hline Diast-CHF \#7 & 2 & 36 & AT & Yes & ASA & 2 \\
\hline Diast-CHF \#8 & 3 & 1160 & $\mathrm{AF}$ & Yes & None & 1 \\
\hline Diast-CHF \#9 & 2 & 9 & AF & No & None & 1 \\
\hline Diast-CHF \#10 & 2 & 13 & AT & Yes & ASA & 5 \\
\hline Find-AF eye \#1 & NA & 1 & AF & No & ASA & 2 \\
\hline Find-AF eye \#2 & NA & 1 & AF & No & None & 4 \\
\hline Find-AF eye \#3 & NA & $>1440$ & AF & Yes & OAC & 4 \\
\hline Find-AF eye \#4 & NA & $>1440$ & AF & Yes & ASA & 4 \\
\hline Find-AF eye \#5 & NA & 989 & AF & Yes & ASA & 4 \\
\hline Find-AF eye \#6 & NA & 215 & AF & Yes & ASA & 3 \\
\hline
\end{tabular}

$\mathrm{AF}$, atrial fibrillation; ASA, acetylsalicylic acid; AT, atrial tachycardia; $\mathrm{CHA}_{2} \mathrm{DS}_{2}$-VASc, congestive heart failure, hypertension, age $\geq 75$ (doubled), diabetes, stroke (doubled)-vascular disease, age 65-74 and sex category (female); NA, not applicable; OAC, oral anticoagulation. 
Table 3 Natriuretic peptide plasma levels in patients with and without paroxysmal AT/AF (5 patients with a history of AF were excluded from the analysis)

\begin{tabular}{lll}
\hline & No paroxysmal AT/AF & Paroxysmal AT/AF \\
\hline Diast-CHF & $n=149$ & $n=8$ \\
NT-proBNP $(\mathrm{pg} / \mathrm{mL})$ & $52.8(30.4-178.0)$ & $154.4(41.7-303.6)$ \\
BNP $(\mathrm{pg} / \mathrm{mL})$ & $43.9(16.3-95.2)$ & $70.0(31.9-142.4)$ \\
Find-AF eye & $n=76$ & $n=6$ \\
NT-proBNP $(\mathrm{pg} / \mathrm{mL})$ & $90.8(42.3-141.7)$ & $216.9(201.4-277.1)$ \\
BNP $(\mathrm{pg} / \mathrm{mL})$ & $29.1(12.0-58.1)$ & $96.0(54.7-108.2)$ \\
NT-proANP (nmol/L) & $1.8(1.0-2.7)$ & $4.4(4.3-4.4)$ \\
\hline All data are displayed as median (25th-75th centile). & & \\
AF, atrial fibrillation; AT, atrial tachycardia; BNP, brain natriuretic peptide; NT-proANP, N-terminal pro-atrial natriuretic peptide, NT-proBNP, \\
N-terminal pro-BNP.
\end{tabular}

\section{Strengths and limitations}

Our study is strengthened by its use and detailed analysis of a continuous 7-day Holter ECG for the evaluation of paroxysmal AF/AT allowed detecting a high number of affected patients, which is higher than that diagnosed by periodic ECGs and cardiac event loop recorders, ${ }^{26}$ and similar findings in two different independent cohorts. Our findings are in line with our prior study in a third cohort. ${ }^{15}$ However, the sample size was too small to perform a detailed analysis on the independence of the indicative value of natriuretic peptide plasma levels for paroxysmal $\mathrm{AT} / \mathrm{AF}$, and the indicative value of $\mathrm{BNP}$ found in the present analysis has to be validated in larger trials. Furthermore, we cannot exclude that more intensive monitoring (eg, by an implantable loop recorder) would have detected a larger number of patients with paroxysmal AF. However, the initial selection of patients with Diast-CHF, which was performed by

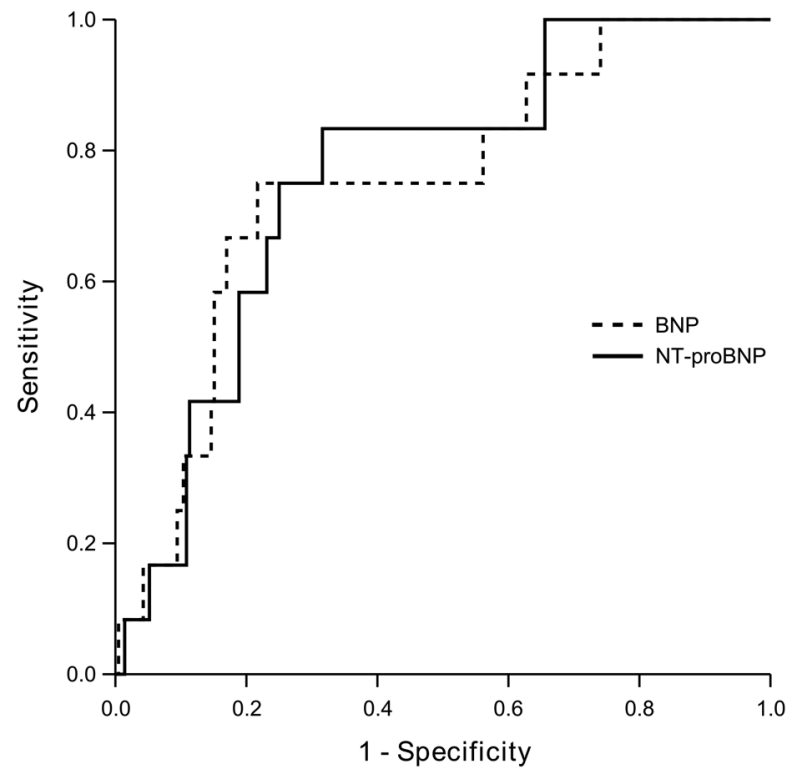

Figure 2 Receiver operating characteristics curve of $\mathrm{N}$-terminal pro-atrial natriuretic peptide plasma levels (dark line) and brain natriuretic peptide (dashed line) for the detection of paroxysmal atrial tachycardia and atrial fibrillation. the lowest and highest quartiles of the baseline NT-proBNP plasma level of the Diast-CHF trial during the baseline visit, may cause an overestimation of the discriminatory value.

The amount of paroxysmal AF which leads to an increase in stroke risk is not known. Although there is now some evidence that patients with episodes lasting longer than $6 \mathrm{~min}$ are at increased risk (ASSERT), ${ }^{17}$ the risk of stroke may already be elevated in patients with excessive supraventricular ectopic activity. ${ }^{27}$ Otherwise, it is not known whether the risk in these patient populations is similar to that in patients with permanent AF and whether these patients benefit similarly from oral anticoagulation.

\section{CONCLUSION}

In two independent patient cohorts, natriuretic peptide levels were higher in patients with $\mathrm{AF} / \mathrm{AT}$ as compared to patients with sinus rhythm. It may therefore be worthwhile to further evaluate the utility of natriuretic peptides in identifying patients at risk for $\mathrm{AF} / \mathrm{AT}$ to be chosen for further workup (eg, prolonged Holter monitoring) as a tool for stroke prevention.

\section{Author affiliations}

${ }^{1}$ Department of Cardiology and Pneumology, University of Göttingen, Göttingen, Germany

${ }^{2}$ Department of Internal Medicine II-Cardiology, University Hospital Regensburg, Regensburg, Germany

${ }^{3}$ Department of Ophthalmology, University of Göttingen, Göttingen, Germany ${ }^{4}$ German Cardiovascular Research Center (DZHK), Göttingen, Germany ${ }^{5}$ Department of Internal Medicine, Cardiology, Charité - Campus VirchowKlinikum, Universitätsmedizin Berlin, Berlin, Germany

${ }^{6}$ German Cardiovascular Research Center (DZHK), partner site Berlin, Germany

${ }^{7}$ Institute for Clinical Epidemiology and Biometry, University of Würzburg, Würzburg, Germany

${ }^{8}$ Department of Clinical Chemistry, University of Göttingen, Göttingen, Germany

${ }^{9}$ Department of Psychosomatic Medicine and Psychotherapy, University of Göttingen, Göttingen, Germany

${ }^{10}$ Department of Neurology, Mainz University Medical Center, Mainz, Germany

Contributors JS and RW wrote the paper. TG collected the data. MZ, AA, MW-K, FE, LB, LB, CH-L, KG, GH, NF and BP provided significant input and improvement of the whole paper. $G G$ performed statistical analysis. 
Funding This study was supported by an unrestricted grant from Roche Diagnostics. This work was supported by grants from the German Federal Ministry of Education and Research (German Heart Failure Network, TP 7 (FKZ 01GI0205)).

Competing interests None declared.

Ethics approval Ethik-Kommission der Universitätsmedizin Göttingen.

Provenance and peer review Not commissioned; externally peer reviewed.

Data sharing statement Consent for publication of raw data not obtained at time of study start (2004).

Open Access This is an Open Access article distributed in accordance with the Creative Commons Attribution Non Commercial (CC BY-NC 4.0) license, which permits others to distribute, remix, adapt, build upon this work noncommercially, and license their derivative works on different terms, provided the original work is properly cited and the use is non-commercial. See: http:// creativecommons.org/licenses/by-nc/4.0/

\section{REFERENCES}

1. Wolf PA, Abbott RD, Kannel WB. Atrial fibrillation as an independent risk factor for stroke: the Framingham Study. Stroke 1991;22:983-8.

2. Hart RG, Pearce LA, Aguilar MI. Meta-analysis: antithrombotic therapy to prevent stroke in patients who have nonvalvular atrial fibrillation. Ann Intern Med 2007;146:857-67.

3. Ziegler PD, Koehler JL, Mehra R. Comparison of continuous versus intermittent monitoring of atrial arrhythmias. Heart Rhythm 2006;3:1445-52.

4. Hohnloser SH, Pajitnev D, Pogue J, et al. Incidence of stroke in paroxysmal versus sustained atrial fibrillation in patients taking ora anticoagulation or combined antiplatelet therapy: an ACTIVE W Substudy. J Am Coll Cardiol 2007;50:2156-61.

5. Stahrenberg R, Weber-Krüger M, Seegers J, et al. Enhanced detection of paroxysmal atrial fibrillation by early and prolonged continuous holter monitoring in patients with cerebral ischemia presenting in sinus rhythm. Stroke 2010;41:2884-8.

6. Jabaudon D, Sztajzel J, Sievert K, et al. Usefulness of ambulatory 7-day ECG monitoring for the detection of atrial fibrillation and flutter after acute stroke and transient ischemic attack. Stroke 2004;35:1647-51.

7. Sanna T, Diener HC, Passman RS, et al. Cryptogenic stroke and underlying atrial fibrillation. N Engl J Med 2014;370(26):2478-86.

8. Engdahl J, Andersson L, Mirskaya M, et al. Stepwise screening of atrial fibrillation in a 75-year-old population: implications for stroke prevention. Circulation 2013;127:930-7.

9. Schnabel RB, Larson MG, Yamamoto JF, et al. Relations of biomarkers of distinct pathophysiological pathways and atrial fibrillation incidence in the community. Circulation 2010;121:200-7.

10. Wazni OM, Martin DO, Marrouche NF, et al. Plasma B-type natriuretic peptide levels predict postoperative atrial fibrillation in patients undergoing cardiac surgery. Circulation 2004;110:124-7.

11. Silvet $\mathrm{H}$, Young-Xu $\mathrm{Y}$, Walleigh $\mathrm{D}$, et al. Brain natriuretic peptide is elevated in outpatients with atrial fibrillation. $\mathrm{Am} J \mathrm{Cardiol}$ 2003;92:1124-7.
12. Nakamura $\mathrm{M}$, Niinuma $\mathrm{H}$, Chiba $\mathrm{M}$, et al. Effect of the maze procedure for atrial fibrillation on atrial and brain natriuretic peptide. Am J Cardiol 1997;79:966-70.

13. Vinch CS, Rashkin J, Logsetty G, et al. Brain natriuretic peptide levels fall rapidly after cardioversion of atrial fibrillation to sinus rhythm. Cardiology 2004;102:188-93.

14. Wozakowska-Kap? $\mathrm{B}$. Effect of sinus rhythm restoration on plasma brain natriuretic peptide in patients with atrial fibrillation. Am J Cardiol 2004;93:1555-8.

15. Wachter R, Lahno R, Haase B, et al. Natriuretic peptides for the detection of paroxysmal atrial fibrillation in patients with cerebral ischemia-the find-AF study. PLOS ONE 2012;7: e34351.

16. Stahrenberg R, Edelmann F, Mende M, et al. The novel biomarker growth differentiation factor 15 in heart failure with normal ejection fraction. Eur J Heart Fail 2010;12:1309-16.

17. Healey JS, Connolly SJ, Gold MR, et al. Subclinical atrial fibrillation and the risk of stroke. N Engl J Med 2012;366:120-9.

18. Camm AJ, Lip GYH, De Caterina R, et al. 2012 focused update of the ESC Guidelines for the management of atrial fibrillation: an update of the 2010 ESC Guidelines for the management of atrial fibrillation-developed with the special contribution of the European Heart Rhythm Association. Europace 2012;14:1385-413.

19. McMurray JJV, Adamopoulos S, Anker SD, et al. ESC guidelines for the diagnosis and treatment of acute and chronic heart failure 2012: the task force for the diagnosis and treatment of acute and chronic heart failure 2012 of the European Society of Cardiology. Developed in collaboration with the Heart Failure Association (HFA) of the ESC. Eur J Heart Fail 2012:14:803-69.

20. Luers $\mathrm{C}$, Wachter R, Kleta S, et al. Natriuretic peptides in the detection of preclinical diastolic or systolic dysfunction. Clin Res Cardiol 2010;99:217-26.

21. Binder L, Pieske B, Olschewski M, et al. N-terminal pro-brain natriuretic peptide or troponin testing followed by echocardiography for risk stratification of acute pulmonary embolism. Circulation 2005;112:1573-9.

22. Patton KK, Ellinor PT, Heckbert SR, et al. N-terminal pro-B-type natriuretic peptide is a major predictor of the development of atrial fibrillation: the Cardiovascular Health Study. Circulation 2009;120:1768-74

23. Solti F, Vecsey T, Kékesi V, et al. The effect of atrial dilatation on the genesis of atrial arrhythmias. Cardiovasc Res 1989;23:882-6.

24. Wachter R, Stahrenberg R, Gröschel K. Subclinical atrial fibrillation: how hard should we look? Heart 2013;99:151-3.

25. Fitzmaurice DA, Hobbs FDR, Jowett $S$, et al. Screening versus routine practice in detection of atrial fibrillation in patients aged 65 or over: cluster randomised controlled trial. BMJ 2007;335:383.

26. Liao J, Khalid Z, Scallan C, et al. Noninvasive cardiac monitoring for detecting paroxysmal atrial fibrillation or flutter after acute ischemic stroke: a systematic review. Stroke 2007;38:2935-40.

27. Binici Z, Intzilakis T, Nielsen OW, et al. Excessive supraventricular ectopic activity and increased risk of atrial fibrillation and stroke. Circulation 2010;121:1904-11.

28. Lip GYH, Frison L, Halperin JL, et al. Identifying patients at high risk for stroke despite anticoagulation: a comparison of contemporary stroke risk stratification schemes in an anticoagulated atrial fibrillation cohort. Stroke 2010;41:2731-8. 